\title{
Classificação da obesidade infantil
}

\author{
Classification of childhood obesity
}

Carlos Alberto Nogueira-de-Almeida ${ }^{1}$, Elza Daniel de Mello², Graziela Aparecida N. de Almeida Ribeiro ${ }^{3}$, Carla Cristina João N. de Almeida ${ }^{4}$, Mário Cícero Falcão ${ }^{5}$, Carla Maria Barreto da Silva de Souza Rêgo ${ }^{6}$

\begin{abstract}
RESUMO
I mportância do Problema: A obesidade na infância e na adolescência é considerada, atualmente, grave problema de saúde pública com prevalência crescente em todo o mundo.

Objetivo: atualizar a classificação da obesidade infantojuvenil publicada originalmente em 2004, incorporando as novas evidências científicas. Métodos: revisão da Literatura Científica que abordou os fatores etiopatogênicos da obesidade, buscando-se aprimorar a classificação anteriormente publicada incluindo os novos conhecimentos científicos. Resultados: apresentação de uma classificação que sugere como primeira estratégia a definição da presença de resistência periférica à insulina e, a seguir, sugere a divisão em quatro subgrupos de obesidade: sindrômica, epigenética, induzida (endocrinológica, neurológica, distúrbios do sono, fármacos e agentes infecciosos) e clássica (somática e psicossocial). Conclusão: a classificação permite auxiliar o clínico na tomada de decisões e na individualização do tratamento.
\end{abstract}

Palavras Chave: Obesidade. Classificação. Criança. Adolescente. Resistência à Insulina. Obesidade Pediátrica.

\begin{abstract}
I mportance of the Problem: obesity in childhood and adolescence is currently considered a serious public health problem with a growing prevalence worldwide. Objective: to update the classification of child and adolescent obesity originally published in 2004, incorporating the new scientific evidence. Methods: a review of the Scientific Literature that addressing etiopathogenic factors of obesity, in order to improve the previously published classification including the new scientific knowledge. Results: a classification that suggests as the first strategy the definition of the presence of peripheral resistance to insulin and then suggests the division into four subgroups of obesity: syndromic, epigenetic, induced (endocrinological, neurological, sleep disorders, drugs and infectious agents) and classical (somatic and psychosocial). Conclusion: this classification could help the clinicians to make decisions and to personalize the treatment.
\end{abstract}

Keywords: Obesity. Classification. Child. Adolescent. Insulin Resistance. Pediatric Obesity.

1. Doutor, Docente, Departamento de Medicina da Universidade Federal de São Carlos (UFSCAR), Brasil

2. Doutora, Docente, Curso de Medicina da Universidade Federal do Rio Grande do Sul, Brasil

3. Doutora, Docente, Centro Universitário Barão de Mauá

4. Especialista em Psicologia, Centro de Investigação em Nutrologia e Saúde, Brasil

5. Doutor, Docente, Faculdade de Medicina da USP, Brasil

6. Doutora, Docente, Faculdade de Medicina da Universidade do Porto, Portugal.
CORRESPONDÊNCIA: Carlos Alberto Nogueira-de-Almeida Rua Eugênio Ferrante, 170

CEP 14027-150 - Ribeirão Preto, SP, Brasil dr.nogueira@me.com

Recebido em 13/11/2017 Aprovado em 30/05/2018

Local onde o trabalho foi desenvolvido: Departamento de Medicina da UFSCAR. 


\section{INTRODUÇÃO}

No ano de 2004, momento em que a epidemia de obesidade começa a ficar evidente em todo o mundo e, paralelamente, cresce o número de publicações científicas sobre o tema, o Centro de Estudos em Saúde e Nutrição Infanto-juvenil da Universidade de Ribeirão Preto (CESNI) publicou uma proposta de classificação clínica, ${ }^{1}$ que foi tema de editorial no mesmo número do periódico. ${ }^{2}$ Posteriormente, ela foi adotada em alguns serviços e incluída em livros de texto. ${ }^{3}$ Àquela época, certos aspectos da classificação, como a importância dada à resistência insulínica, eram bastante inovadores e, até mesmo, polêmicos, conforme se pode verificar à leitura do editorial escrito por Damiani. ${ }^{2}$ Basicamente, o que se propôs naquele momento foi que o profissional que atende a criança obesa busque levantar todos os possíveis quadros que pudessem atuar como disparadores ou mantenedores da adiposidade excessiva. De acordo com os conhecimentos disponíveis à época, propôs-se que classificação em dois grandes grupos: com e sem resistência periférica à insulina. O primeiro foi chamado de Obesidade Metabólica. O outro grupo foi subdividido em Obesidade Sindrômica, Obesidade Induzida Endocrinológica, Obesidade Induzida Neurológica, Obesidade Induzida por Fármacos, Obesidade Alimentar Somática e Obesidade Alimentar Psicossomática

Por outro lado, fatores hoje bem estudados, como a programação metabólica, a microbiota, as infecções virais e a importância do sono, eram bem menos conhecidos. Em 2010, Han e colaboradores ${ }^{4}$ publicaram no periódico Lancet uma proposta de classificação bastante semelhante à anteriormente sugerida em 2004 por nosso grupo. Esses autores também se basearam fundamentalmente na ideia de que a obesidade é uma doença de origem variada, podendo estar associada a: medicações obesogênicas, doenças hormonais (hipotireoidismo, deficiência de hormônio de crescimento, doença de Cushing e pseudohipoparatireoidismo), alterações hipotalâmicas, quadros genéticos (Pradder-Willi, Bardet-Biedl, entre outros) e problemas relacionados ao estilo de vida (dieta e atividade física).

Atualmente, os números da obesidade são impactantes e sua prevalência aumentou substancialmente nas últimas três décadas, tanto nos países desenvolvidos como naqueles em desenvolvimento. ${ }^{4}$ Estima-se que no mundo 41 milhões de crianças menores de cinco anos estejam obesas. ${ }^{5} \mathrm{O}$ Brasil enfrenta de forma evidente um processo de transição nutricional. Ao mesmo tempo em que a desnutrição energético-proteica tem apresentado queda em sua prevalência, o sobrepeso e a obesidade rapidamente se candidatam ao posto de maior problema nutricional do país.

Apesar de não existirem dados atualizados, a última Pesquisa de Orçamentos Familiares ${ }^{6}$ mostrou que, para as crianças com idades entre cinco e nove anos, a prevalência de sobrepeso/obesidade saltou, entre 1989 e 2009, de 13,8\% (meninos) e $10,4 \%$ (meninas) para $51,4 \%$ e $43,8 \%$, respectivamente e, entre os adolescentes, de $20,8 \%$ (meninos) e $18,1 \%$ (meninas) para $27,6 \%$ e $23,4 \%$, respectivamente.

Para a criança, a obesidade representa, muitas vezes, o fator desencadeante para uma série de comorbidades que interferem na saúde atual e podem persistir até a vida adulta. Essas comorbidades, que antes se acreditavam presentes apenas nos adultos, já são demonstradas em crianças e adolescentes, aparecendo já na fase de sobrepeso, ${ }^{7} \mathrm{em}$ todas as classes sociais. ${ }^{8}$ Dentre elas, destacam-se $\mathrm{e}^{4,8-11}$ : dislipidemias, resistência insulínica, problemas ortopédicos, hipertensão arterial, esteatose hepática, modificações da geometria cardiovascular, alterações hepáticas, aumento da espessura carotídea, entre outras.

A abordagem terapêutica da obesidade é bastante desafiadora, especialmente para o profissional que atua em ambulatório. Uma das formas de otimizar o tratamento é classificar adequadamente cada caso. Pacientes obesos tem em comum o aumento da adiposidade corporal, que pode ser entendido como um aspecto fenotípico de expressão comum a numerosos quadros clínicos que, de alguma forma, promovem balanço energético positivo. Mas é certo que, por exemplo, uma criança que acumulou gordura excessiva devido ao uso de um fármaco obesogênico será tratada de forma distinta daquela que apresenta uma síndrome genética.

O presente artigo descreve uma classificação etiopatogênica da obesidade e incorpora e atualiza a proposta anteriormente publicada, ${ }^{1}$ mantendo o mesmo objetivo de ser uma ferramenta para a tomada de decisão terapêutica. 
A figura 1 mostra, de forma esquemática a classificação e, a seguir, serão discutidos cada um de seus tópicos.

\section{MATERIAL E MÉTODOS}

A presente classificação tem sua origem principal na experiência de seus autores nos serviços em que atuam. O embasamento teórico dessas práticas fundamenta-se em bibliografia científica constantemente atualizada e que, no presente artigo, foi obtida nas bases de dados Scielo e Pubmed, nas línguas portuguesa, espanhola e inglesa, referentes ao período compreendido entre 1980 e 2018.

\section{Excesso de adiposidade corporal}

A primeira etapa de classificação da criança passa pela confirmação de que, efetivamente, trata-se de obesidade. O passo inicial é a utiliza-

\section{Excesso de adiposidade corporal}

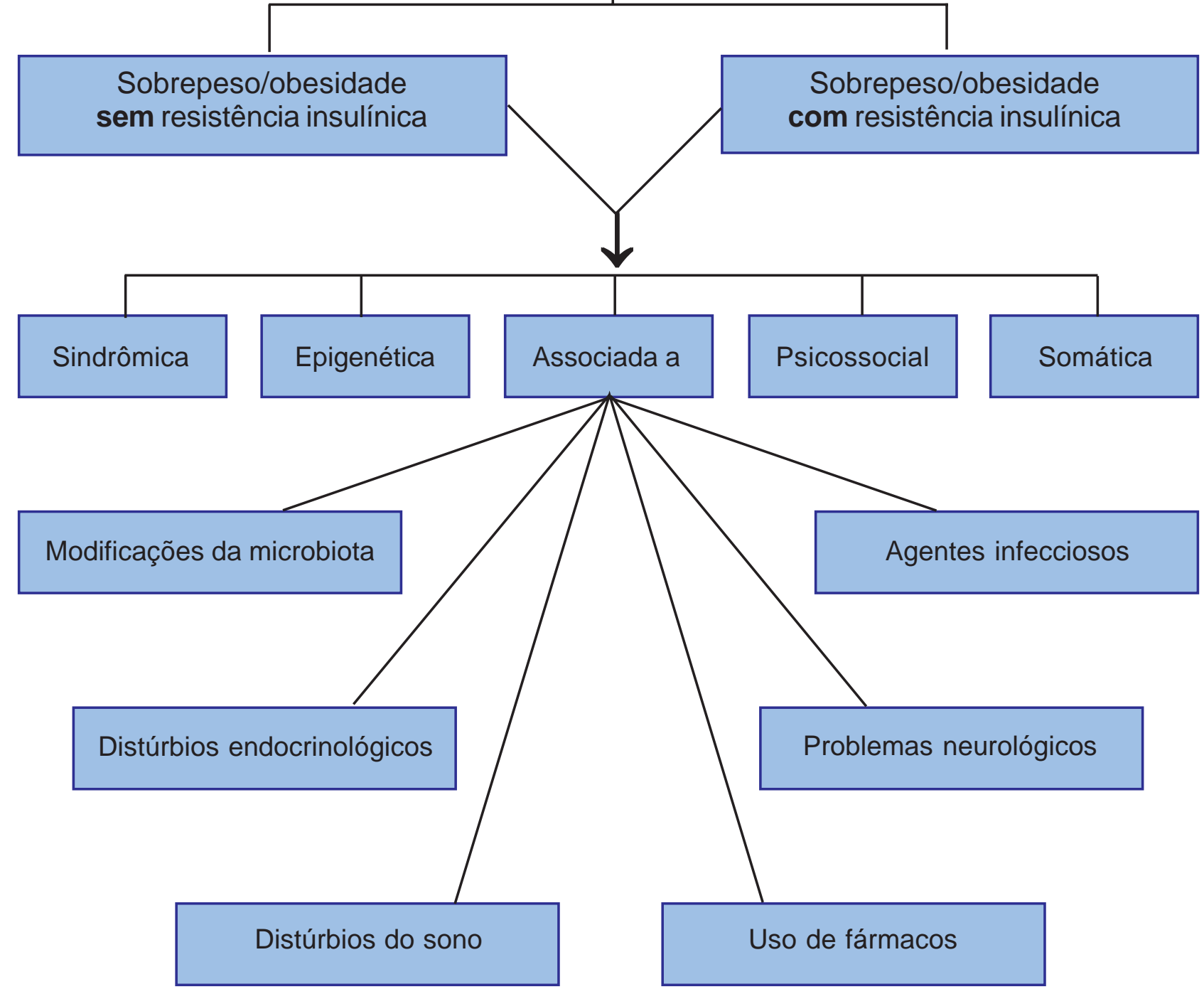

Figura 1. Classificação da obesidade. 
ção de peso e estatura para cálculo do índice de massa corporal (IMC) e plotagem na curva de escores $z .{ }^{12}$ Valores acima do escore $z+2$ para crianças abaixo de 5 anos ou +1 para aquelas com mais de 5 anos devem ser considerados elevados, mesmo que configurem apenas sobrepeso, uma vez que as comorbidades já aparecem nessa fase. ${ }^{7}$

Considerando-se que, nem todo excesso de peso significa aumento da adiposidade, é importante confirmar o diagnóstico com algum tipo de avaliação de composição corporal, podendo-se utilizar o exame físico ou medidas específicas, tais como circunferência abdominal, dobras cutâneas, bioimpedanciometria ou outros métodos de avaliação corpórea. ${ }^{12}$

\section{Sobrepeso/obesidade com resistência insulínica}

O aumento do índice de massa corporal correlaciona-se fortemente com o agravamento da resistência insulínica ${ }^{13}$ e, apesar da dificuldade em diagnosticar essa condição na infância, ${ }^{14}$ especialmente do ponto de vista clínico, dois parâmetros podem ser usados ${ }^{15}$ : a determinação da insulinemia de jejum e o cálculo de homeostasis model assessment (HOMA-IR). Em ambos os casos, a informação obtida será semelhante, uma vez que, na faixa etária pediátrica, a glicemia apresenta pouca variação e o HOMA-IR leva em conta, justamente, insulinemia e glicemia. ${ }^{14}$ Ainda não foram definidos valores de corte internacionais em relação à insulinemia de jejum e HOMAIR para a faixa etária pediátrica. Desde a publicação de Tem e McLaren, ${ }^{16}$ o valor de $15 \mathrm{microUl} / \mathrm{mL}$ tem sido usado, mas ainda sem validação. Outros pontos de corte, como 12, 27 e 30 também já foram sugeridos. ${ }^{17}$

Durante a puberdade, ocorrem eventos que se assemelham a uma "resistência insulínica fisiológica". ${ }^{18,19,20}$ Sendo assim, seria imperativo que os pontos de corte fossem definidos respeitando-se a variação biológica desse indicador. Recentemente, Cuartero e colaboradores ${ }^{21}$ propuseram o uso do percentil 90 baseado em levantamento que incluiu 372 indivíduos com idades entre um mês e 18 anos, no qual observaram variação importante desses indicadores em relação ao estadiamento puberal, idade e sexo. Ape- sar de bastante adequado, do ponto de vista técnico, a utilização prática, para estudos populacionais, esbarra na dificuldade da avaliação dos critérios de Tanner de todos os pacientes envolvidos.

Estudo anterior, ${ }^{22}$ conduzido apenas com escolares e adolescentes eutróficos, mostrou que a insulinemia de jejum varia de maneira significativa entre as idades propostas, de forma a pressupor a necessidade de uma curva, com valores ascendentes até os 13-15 anos, com posterior queda, tanto para meninos como para meninas. Sendo assim, de acordo com recente publicação de nosso grupo, ${ }^{23}$ sugere-se que a insulinemia de jejum seja o critério utilizado na prática clínica para definição de resistência insulínica, com os seguintes pontos de corte:

$\Rightarrow 7$ a 8,9 anos: $7,92 \mu \mathrm{U} / \mathrm{mL}$ (meninos) e $6,70 \mu \mathrm{U} / \mathrm{mL}$ (meninas)

$\Rightarrow 9$ a 10,9 anos: $8,69 \mu \mathrm{U} / \mathrm{mL}$ (meninos) e $11,99 \mu \mathrm{U} / \mathrm{mL}$ (meninas)

$\Rightarrow 11$ a 12,9 anos: $12,18 \mu \mathrm{U} / \mathrm{mL}$ (meninos) e $13,26 \mu \mathrm{U} / \mathrm{mL}$ (meninas)

$\Rightarrow 13$ a 14,9 anos: $13,74 \mu \mathrm{U} / \mathrm{mL}$ (meninos) e $14,85 \mu \mathrm{U} / \mathrm{mL}$ (meninas)

$\Rightarrow 15$ a 17,9 anos: $10,27 \mu \mathrm{U} / \mathrm{mL}$ (meninos) e $13,13 \mu \mathrm{U} / \mathrm{mL}$ (meninas)

Mais importante que o simples diagnóstico da resistência periférica à insulina, justifica-se sua categorização como primeira etapa, dadas as consequências para a saúde da criança, que nem sempre estão correlacionadas à gravidade do excesso de peso e aparecem, muitas vezes, de forma precoce, mesmo nos casos mais leves. ${ }^{24}$

O exame físico revela predominância de adiposidade nas regiões abdominais e cervical posterior, estatura elevada e acantose nigricans 4 . A maior parte das comorbidades se apresenta de forma mais prevalente ou, pelo menos, mais grave, na presença de resistência insulínica. ${ }^{25}$ Podemse destacar: diabetes, dislipidemia, hipertensão arterial, síndrome dos ovários policísticos, doença hepática gordurosa, aceleração da puberdade e estrias. ${ }^{25}$ Por fim, é importante destacar o efeito "círculo vicioso", uma vez que a obesidade precipita e agrava a resistência periférica à insulina 
a qual, por sua vez, devido possivelmente à hiperinsulinemia, leva a processo anabólico, com aumento do apetite e da adipogênese. ${ }^{26}$ Esse último aspecto pode ser, inclusive, demonstrado por um ensaio clínico recente que utilizou como intervenção única a prescrição de metformina (que atua reduzindo a resistência insulínica) para crianças obesas, obtendo-se emagrecimento, mesmo na ausência de outras medidas, como dieta ou aumento da atividade física. ${ }^{27}$ Devido às maiores complexidade e gravidade dessa forma de apresentação da obesidade, o tratamento é sempre mais difícil e demorado.

\section{Sobrepeso/obesidade sem resistência insulínica}

Nesses casos, apesar de quase sempre presentes, as comorbidades são menos frequentes e graves, sendo que crianças com escores z maiores tendem a apresentar mais problemas associados. ${ }^{28}$ Por outro lado, nesse grupo, encontram-se muitas crianças que, excetuando-se o excesso de peso, parecem absolutamente normais. Muitos pacientes têm melhor adesão ao tratamento convencional, respondendo de forma mais rápida às medidas terapêuticas.

A segunda etapa da classificação, mostrada a seguir, aplica-se aos dois casos descritos acima, sugerindo-se que, para efeito de denominação, sejam utilizados todos os aspectos, por exemplo, “obesidade com resistência insulínica associada a distúrbio do sono" ou ainda "obesidade psicossocial sem resistência insulínica".

\section{Obesidade sindrômica}

Sabe-se que a obesidade, de uma maneira geral, apresenta forte componente genético, sendo que muitos autores consideram que se trata de uma doença de etiologia poligênica clássica, em que o fenótipo final será a resultante da combinação entre diversos genes. ${ }^{29,30}$

Já foram, por exemplo, identificados genes envolvidos em baixa taxa de metabolismo basal, baixo efeito termogênico dos alimentos, baixo grau de oxidação das reservas gordurosas, altos níveis de atividade da lipase lipoproteica, baixos graus de mobilização lipídica, inadequada regulação central de fome/saciedade, baixo grau oxidativo da musculatura esquelética, dentre outros. ${ }^{29,30}$
Por outro lado, a obesidade pode aparecer também como resultado de diversas doenças genéticas mais específicas, em que apenas um defeito encontrado é suficiente para predispor à adiposidade excessiva.

O exemplo mais clássico é a mutação no gene OB que leva à deficiência na produção de leptina, principal hormônio responsável pelo controle do balanço energético. Trata-se de quadro bastante raro, mas dramático, na medida em que a obesidade resultante é extremamente grave ${ }^{31}$. Deve-se citar também o gene FTO, já bastante estudado, onde indivíduos com determinadas variantes apresentam quadro de obesidade clássica. ${ }^{32}$

Outras síndromes genéticas cursam com obesidade, ${ }^{33}$ tais como: síndrome de Prader Wili, Bardet-Biedl, Alström, WAGR, Cohen, Alstrom, Borjeson-Forssman-Lehmann, BeckwithWiedemann e Down. Observa-se associação com malformações congênitas (leves ou graves), dismorfias faciais, distúrbios de crescimento (hiper ou hipocrescimento), atraso de desenvolvimento neuro-psicomotor, deficiência mental, história familiar sugestiva, lembrando que a ausência de casos familiares não afasta o fator genético e consanguinidade. ${ }^{34}$

A história de excesso de peso pode ser neonatal (onde devem ser avaliadas as síndromes de hipercrescimento) ou aparecer com o avançar da idade. ${ }^{34}$ No exame físico, encontram-se malformações leves e/ou graves, dismorfias faciais, baixa ou alta estatura e alterações neurológicas. ${ }^{34}$

No caso específico da Síndrome de PraderWilli, 33,35 encontram-se hipotonia e dificuldades de alimentação no primeiro ano de vida e, posteriormente, aparecem hipogonadismo, retardo mental, redução na velocidade de crescimento e hiperfagia intensa ${ }^{35}$. O controle da obesidade pode ser difícil, quando a hiperfagia de origem central está presente, como na síndrome de Prader-Willi, ou a hipotonia, no caso da síndrome de Down, que reduz o gasto calórico basal ${ }^{36}$. Além disso, a deficiência mental pode atuar como um fator que interfere no controle da dieta ${ }^{36}$. Na maioria das vezes, a doença de base não pode ser tratada e a abordagem da obesidade deve ser feita por educação do paciente e da família, restrição dietética, exercícios físicos e, eventualmente, medicamentos que, em geral, tem pouca efetividade. 


\section{Obesidade epigenética}

Programação metabólica é definida como o resultado de "modificações não estruturais do material genético, consequentes de insulto ou estímulo aplicado em um período crítico ou sensível, com potencial de efeito duradouro ou persistente sobre a estrutura ou a função de um organismo". 37

O mecanismo genético de programação metabólica é a epigenética, que é definida como "todas as mudanças reversíveis e herdáveis no genoma funcional que não alteram a sequência de nucleotídeos do DNA". ${ }^{38}$ Há três mecanismos epigenéticos principais: a metilação, as mudanças químicas nas histonas e a atuação dos RNAs não-codificantes. ${ }^{39}$

A metilação é um processo onde radicais metil são adicionados a trechos específicos da molécula de DNA, alterando a expressão de determinado gene. As histonas são estruturas em volta das quais o DNA se organiza e, modificações em sua configuração espacial, são capazes de controlar a transcrição. Por fim, os RNAs nãocodificantes são pequenas fitas de nucleotídeos capazes de modular a expressão dos genes por meio do silenciamento transcricional ou pós-transcricional.

A demonstração mais evidente de programação metabólica ocorre com a obesidade, ${ }^{38}$ uma vez que fetos submetidos à subnutrição intrauterina conseguem se adaptar para sobreviver, mas, para isso, são reprogramados para serem conservadores de energia. ${ }^{40}$ Após o nascimento, se a restrição alimentar cessa, o novo perfil fenotípico leva ao acúmulo excessivo de gordura. ${ }^{41}$

Os estudos posteriores às demonstrações de Barker ${ }^{40,42,43}$ mostraram que não se tratava apenas de uma simples habilidade para conservar energia, mas a todo um conjunto de mudanças no perfil metabólico, essenciais na fase intrauterina, mas inadequadas no meio externo. ${ }^{44}$ Várias dessas mudanças acabam repercutindo na vida adulta, 45 gerando diversos quadros, tais como: maior pressão arterial, risco elevado de diabetes, dislipidemias, redução na densidade óssea, respostas inadequadas ao estresse, menor elasticidade arterial, alterações de perfis hormonais e, até mesmo, maior propensão a algumas doenças psíquicas ${ }^{46}$.
Observou-se também que não somente a subnutrição intrauterina poderia reprogramar os genes, mas vários outros agravos, nutricionais ou não, em períodos críticos, de elevada plasticidade, como os primeiros 1000 dias de vida (período gestacional mais os dois primeiros anos de vida). ${ }^{47}$

Hoje se reconhece que muitos aspectos ligados à saúde da mãe, podem, adicionalmente, interferir nessa programação. ${ }^{48}$ Hofman e colaboradores $^{49}$ propõe que o ambiente adverso, que leva ao insulto inicial capaz de fazer a programação metabólica, ocorre de forma diferente de acordo com o perfil de nascimento. Nas crianças de termo, mas com restrição de crescimento intrauterino, o agravo ocorreria na fase pré-natal; por outro lado, no caso dos prematuros, aconteceria nos primeiros meses de vida. De fato, vários estudos têm mostrado que o perfil pós-natal de crescimento e ganho de peso acelerados observados em crianças nascidas prematuramente, mas também em crianças de termo quando alimentados inadequadamente, leva a diminuição da sensibilidade à insulina, alterações de padrões de crescimento e aumento da adiposidade. ${ }^{39}$

Cabe destacar que um dos fatores mais relevantes, que leva a crescimento pós-natal acelerado e obesidade futura é o excesso de consumo de proteínas nos primeiros meses de vida, ${ }^{50}$ sendo esse um exemplo clássico de como se pode atuar na prevenção da obesidade, estimulando o aleitamento materno ou, na impossibilidade deste, prescrevendo fórmulas infantis com teor proteico adequado e evitando-se o uso de leite de vaca não modificado.

Muitas vezes, a procura por explicações quando a obesidade já se encontra instalada é difícil e leva a resultados surpreendentes. Nogueira-de-Almeida e colaboradores, ${ }^{51}$ em artigo publicado em 2015, mostraram que a ingestão calórica e o padrão de atividade física eram semelhantes quando crianças obesas eram comparadas a seus pares eutróficos. Sendo assim, parece que a busca pelos fatores causais precoces pode ser a chave para a melhor compreensão da obesidade.

Nesse sentido, o reconhecimento da programação metabólica, que pode ocorrer no começo da vida, é fundamental para que todas as medidas sejam tomadas, tanto do ponto de vista de saúde pública, como no atendimento individual, para que a criança possa viver em um ambien- 
te adequado. Do ponto de vista nutricional, esse trabalho começa com a atuação do pediatra antes do nascimento, junto à gestante. ${ }^{52}$ Depois, dentro da proposta da Puericultura, inclusive nos programas de saúde da família, ${ }^{53}$ com o estímulo ao aleitamento materno e, enfim, completa-se com a adequada orientação à família sobre fórmulas infantis, alimentação complementar, suplementação de micronutrientes e importância da atividade física.

\section{Obesidade associada a modificações de microbiota}

A microbiota intestinal pode afetar o peso corporal. Este aspecto é muito estudado em animais e acredita-se que o mecanismo seja semeIhante nos seres humanos, ou seja, efeitos sobre o metabolismo energético, grau de inflamação e alteração da permeabilidade intestinal. ${ }^{54,55}$

Recentemente vários pesquisadores têm sugerido que existe relação entre as bactérias residentes no trato intestinal e o potencial de ganho de peso. Estudos em animais sugerem que a administração de antibióticos no início da vida predispõe à obesidade mais tarde, ${ }^{56}$ mas existem estudos observacionais em humanos que também apoiam esta possibilidade. ${ }^{57-60} \mathrm{O}$ estudo de Gerber et al, ${ }^{61}$ que inclui mais de 142.000 crianças, encontrou associação persistente, progressiva e reversível entre o uso de antibióticos e subsequentes alterações do IMC. Portanto, este é mais um motivo para o uso racional de antibióticos em pediatria: prevenir alteração de flora infantil e o desenvolvimento de obesidade.

Também são encontrados estudos em modelos animais que sugerem que modificações induzidas na microbiota intestinal podem ser responsáveis por alguma perda de peso e melhorias na sensibilidade à insulina, especialmente após a cirurgia de bypass gástrico. ${ }^{62}$ Ainda não está claro se esses mecanismos são relevantes para a gênese da obesidade e mesmo sua epidemia em humanos. Obviamente, não se trata de indicar ou supervalorizar as cirurgias, mas sim de chamar atenção para o sucesso de pesquisas com probióticos e mesmo transplantes de fezes, já usados para o manejo da obesidade.

Outros estudos em animais sugerem que certas misturas de bactérias residentes induzem o desenvolvimento de gordura marrom ${ }^{63}$ e já foi demonstrado que, na dependência do perfil da microbiota, pode ocorrer recuperação de calorias da fibra dietética através de fermentação em ácidos graxos de cadeia curta. ${ }^{64}$

\section{Obesidade associada a distúrbios endocrinológicos}

Trata-se de quadro de obesidade associado a doenças do sistema endócrino como hipotireoidismo, hipercortisolismo, pseudohipoparatireoidismo, deficiência de hormônio de crescimento e síndrome dos ovários policísticos (SOP). Muitas vezes não é possível definir relação de causa e os quadros, quando diagnosticados, podem aparecem juntos. ${ }^{65}$ De qualquer forma, mesmo que não possa ser considerado como fator etiológico, o distúrbio endocrinológico deverá ser adequadamente abordado para que não se configure como elemento perpetuador da obesidade. ${ }^{65}$

O quadro clínico depende da doença de base e um dos principais achados é a baixa estatura, comum à maior parte das doenças endocrinológicas que cursam com obesidade. ${ }^{66}$ É importante lembrar que, muitas vezes, a própria obesidade aparece como o primeiro sintoma a levar a família a procurar ajuda. Nesses casos, é de extrema importância que o clínico esteja atento à história, levando em conta uma série de sintomas que podem revelar a doença de base.

Nos casos de hipotireoidismo, pode-se encontrar mixedema, atraso da idade óssea, letargia, pele seca, bradicardia e bradipnéia. ${ }^{67} \mathrm{O}$ hipercortisolismo leva a hirsutismo, fácies cushingóide, distribuição centrípeta da adiposidade, estrias, acne, pletora, osteoporose e exacerbação de caracteres masculinos. ${ }^{68} \mathrm{O}$ pseudohipoparatireoidismo cursa com resistência à ação do paratormônio, anomalias dentárias, pele seca, unhas quebradiças e tetanias. ${ }^{69} \mathrm{~A}$ deficiência de hormônio de crescimento mostra basicamente baixa estatura evidente e redução na velocidade de crescimento. ${ }^{70}$ A SOP é doença exclusiva de meninas e ocasiona hirsutismo, distúrbios menstruais, acne e alopecia, ${ }^{71}$ sendo que a ocorrência de hiperinsulinismo e resistência periférica à insulina é frequente. ${ }^{72}$

A etapa primordial do tratamento é o controle da doença de base, com reposição hormonal 
nos casos de hipotireoidismo e deficiência de hormônio de crescimento. O controle do hipercortisolismo depende da etilogia, podendo ser cirúrgico nos casos dos adenomas. A SOP necessita de tratamento apropriado que permita a ovulação adequada, sendo que, nos casos em que há concomitância de resistência periférica à insulina, a metformina tem sido utilizada com resultados satisfatórios. ${ }^{73}$

A relação entre hormônios e obesidade é bastante complexa. Muitas doenças genéticas (tais como a deficiência congênita de leptina) apresentam interface hormonal evidente. De igual forma, é provável que muitas crianças obesas apresentem defeitos de produção ou de sinalização de hormônios ainda não completamente estudados, mas sabidamente ligados aos processos de fome, saciedade e balanço energético, tais como grelina, peptídeo YY (PYY), pró-ópio-melanocortina (PONC), transcrito regulador de cocaína e anfetamina (CART), neuropeptídio Y (NPY), peptídeo relacionado ao gene agouti (AgRP), hormônio concentrador de melanina $(\mathrm{MCH})$, orexina, galanina, ácido gama-aminobutírico (GABA), entre outros. ${ }^{66} \hat{A}$ medida que distúrbios endocrinológicos específicos desses hormônios forem descobertos, é possível que, dentro da classificação proposta, passem a figurar no presente tópico. Entretanto, até a presente data, isso ainda não é possível.

\section{Obesidade associada a problemas neurológicos}

A obesidade de origem hipotalâmica é doença rara em seres humanos. Ocorre quando existe lesão na região ventromedial ou paraventricular do hipotálamo ou na amígdala cerebral. Essas regiões do cérebro são responsáveis pela integração de informações metabólicas sobre reservas de nutrientes com impulsos sensoriais aferentes sobre a disponibilidade de alimentos.

Quando ocorre lesão, podem causar alterações metabólicas, como hiperfagia, hiperinsulinemia, prejuízo da termogênese dos alimentos, além de desordens funcionais no sistema nervoso autônomo; esses aspectos estão associados com a gênese de obesidade. Esta condição pode ser causada por trauma, tumor, doença inflamatória, cirurgia de fossa posterior ou aumento da pressão intracraniana. ${ }^{74}$
Em Pediatria, esta condição pode ser observada especialmente em pacientes com craniofaringioma, um tumor embrionário localizado na região do hipotálamo/hipófise, que causa danos ao hipotálamo devido ao próprio tumor ou pelo tratamento (excisão cirúrgica e radioterapia). É uma neoplasia de natureza benigna, pouco frequente, responsável por $1 \%$ a $3 \%$ de todos os tumores intracranianos.

Outros fatores também podem fazer parte da etiologia da obesidade hipotalâmica ${ }^{74}$, tais como: tumores (epitelioma, angiosarcoma, colesteatoma, pinealoma, greminoma, endotelioma, ependimoma, glioma, meningioma, macroadenoma hipofisário, teratoma, metástases); inflamação (sarcoidose, tuberculose, aracnoidite, histiocitose $X$, encefalites); síndromes genéticas (síndrome de Prader-Willi, síndrome de Bardet-Biedl); traumatismo craniano; neurocirurgia; radioterapia craniana; aneurisma cerebral; drogas psicotrópicas.

O mecanismo proposto para a obesidade hipotalâmica tem sido descrito como um desequilíbrio da ação de hormônios orexígenos e anorexígenos decorrentes dos fatores acima mencionados, que levam à sinalização hipotalâmica disfuncional da homeostase energética. Embora alguns estudos tenham demonstrado que estratégias terapêuticas não farmacológicas, como dieta e atividade física, estejam associadas com melhora na composição corporal e retardo no ganho de peso, ainda são necessários mais estudos para estabelecer a terapia mais adequada para esses indivíduos. ${ }^{75}$

\section{Obesidade associada a distúrbios do sono}

Há algum tempo já é reconhecida a importante influência do sono na gênese e na manutenção da obesidade. Revisão sistemática de 25 estudos publicados entre 2006 e 2011 envolvendo crianças e adolescentes entre zero e 19 anos de idade, verificou que $100 \%$ dos estudos mostravam associação estatisticamente significativa entre curta duração do sono e obesidade. ${ }^{76}$

Acredita-se que problemas de sono possam estar relacionados à obesidade desde o início da vida. ${ }^{77}$ Vários fatores são reconhecidos como causadores desse efeito, tais como: aumento da fome; aumento da oportunidade de comer, termorregulação prejudicada, maior fadiga, aumen- 
to dos hormônios ligados ao estresse e aumento da produção de grelina. ${ }^{78}$

É bastante difícil estabelecer a quantidade de sono ideal para uma criança, sendo reconhecido o fato de haver grande variação individual. Por outro lado, sabe-se que o peso abdominal da criança obesa exerce, na posição horizontal, forte pressão sobre o diafragma, que é o principal músculo respiratório, fazendo com que ele diminua sua capacidade de trabalho. Esse fato leva à dificuldade respiratória importante, com períodos curtos de completa parada na respiração, conhecidos como apneia. Esses períodos, apesar de sutis, fazem a criança acordar dezenas de vezes no decorrer da noite, dificultando o aprofundamento do sono. Muitas vezes a irritabilidade e a sonolência diurna observadas em algumas crianças obesas devem-se a esse fato. ${ }^{79}$

Sendo assim, fica claro que a relação entre sono e obesidade ocorre por um círculo vicioso, em que os dois quadros se retroalimentam, tornando o tratamento ainda mais difícil, uma vez que os mecanismos desse processo ainda não são totalmente conhecidos $^{80}$.

\section{Obesidade associada ao uso de fármacos}

É um quadro desencadeado e/ou mantido pela utilização de fármacos atuando como orexígenos, agravando a resistência insulínica, aumentando a deposição de gordura ou reduzindo o metabolismo basal. Em geral, a anamnese revela a relação entre o uso do fármaco e o surgimento ou o agravamento da obesidade.

Os fármacos habitualmente envolvidos são: corticoides, antipsicóticos (valproato de sódio, clozapina, olanzapina, risperidona, quetiapina, clorpromazina), antidepressivos (amitripitilina, imipramina, nortriptilina), antihistamínicos, estabilizadores do humor (carbonato de lítio, ácido valpróico e carbamazepina), anticonvulsivantes (gabapentina, carbamazepina), alguns antineoplásicos, insulina, secretagogos de insulina, antihipertensivos (propranolol, clonidina) e contraceptivos. ${ }^{66,81-84}$

No exame físico podem-se observar sinais relativos à doença que levou à utilização do fármaco ou sinais causados pelo próprio medicamento. A suspensão ou substituição da droga está sempre indicada, apesar de não ser sempre possível. Em alguns casos, têm-se sugerido a asso- ciação de outros fármacos que antagonizem o efeito obesogênico, como o uso de topiramato ou metformina, em pacientes em tratamento de esquizofrenia ou outras doenças psiquiátricas. ${ }^{81,83,85}$

\section{Obesidade associada a agentes infecciosos}

Alguns agentes infecciosos já foram associados com a obesidade, especialmente o adenovírus. ${ }^{86,87}$ Estudos em humanos, incluindo um pequeno ensaio em gêmeos, mostrou associação entre anticorpos para o adenovírus 36 e estado de obesidade em adultos, mas essa associação não foi confirmada em outros estudos. ${ }^{88,89}$ Possíveis explicações para as observações em humanos incluem uma verdadeira associação causal, a vulnerabilidade à infecção por adenovírus ou a presença de fatores de confusão não medidos.

Em estudo transversal de 124 escolares e adolescentes com idade entre oito e 18 anos, 90 os anticorpos para o subgrupo $D$ do adenovírus tipo 36 foram encontradas em 19 indivíduos. Quinze $(78 \%)$ dos 19 indivíduos soropositivos eram obesos (IMC $\geq$ percentil 95) e a presença destes anticorpos foi significativamente mais frequente entre indivíduos obesos que entre os não obesos ( 22 versus $7 \%$ ); entre as crianças obesas, aquelas que eram soropositivas tinham maiores peso, IMC, circunferência da cintura e relação cintura-altura quando comparadas com as seronegativas. Embora estes resultados sugiram possível ligação entre o adenovirus 36 e a obesidade, ainda não foi definitivamente estabelecida uma relação causal, mas apenas associação.

\section{Obesidade psicossocial}

A obesidade nestes casos está associada ao aumento da ingestão de alimentos diante de estados emocionais diversos. A fim de tentar explicar este fato, primeiramente é preciso pontuar a importância da alimentação durante a infância, bem como a relação entre mãe/cuidador e a criança.

Ao nascer, mais do que ser alimentado fisicamente e consequentemente ter suas necessidades nutricionais satisfeitas, a criança necessita receber afeto, atenção e cuidado, o que favorecerá o estabelecimento do vínculo entre mãe/ cuidador e criança, suprindo assim suas necessi- 
dades emocionais. À medida em que esse vínculo vai se construindo de maneira inadequada ou conflituosa, a criança pode se tornar mais vulnerável ao desenvolvimento de doenças, entre elas a obesidade. Neste caso, pode ocorrer, por exemplo, superalimentação por parte da mãe/cuidador em função da sua dificuldade em distinguir a fome da criança de outras necessidades.

Assim, ao invés de oferecer atenção, afeto, carinho, cuidado e acolhimento necessários ao filho, são oferecidos alimentos como tentativa de suprimir o desconforto da criança. Esta aprende assim que, diante de conflitos e dificuldades o alimento minimiza o sofrimento emocional. ${ }^{91,92}$

A Teoria do Apego de Bowlby ${ }^{93}$ oferece suporte à hipótese anteriormente destacada na medida em que aponta que todo ser humano busca a proximidade de pessoas significativas diante de situações de ameaça ou de necessidades diversas. Ao se aproximar dessas pessoas e obter o cuidado e o afeto necessários, o indivíduo tenderia a se sentir mais seguro. Ao contrário, quando não há disponibilidade dessas figuras significativas ou quando essas não são capazes de oferecer o suporte necessário ou adequado, podem ocorrer dificuldades em lidar com tais situações de ameaça, insegurança e necessidade, predispondo o indivíduo ao desenvolvimento de doenças como a obesidade. Neste caso, o alimento é utilizado como forma de minimizar os conflitos emocionais.

Com base nesta teoria, destaca-se a importância de figuras representativas para a criança, podendo ser os pais ou os cuidadores. Nos dias de hoje, com as mudanças que estão sendo vivenciadas na família, além do tradicional papel da mãe, o pai têm surgido como pessoa significativa nos cuidados com os filhos, alcançando lugar de destaque na conformação familiar, colocandose como figura emocionalmente disponível, sensível e que promova segurança, favorecendo o aprendizado de estratégias de autorregulação emocional e de respostas adequadas aos problemas psicológicos, contribuindo para minimizar os riscos da obesidade psicossocial. ${ }^{94}$

Apesar de ser reconhecida a importância da proximidade de pessoas significativas, como anteriormente salientado, existem características psíquicas individuais de cada pessoa.
Assim, as necessidades de afeto e atenção vão encontrar respaldo nas mais diversas formas. A experiência vivenciada na prática clínica, quando se pensa em obesidade, permite listar uma série de combinações e possibilidades, que se materializam em alguns esteriótipos, amplamente observados, sendo os mais comuns descritos abaixo $95,96,97$ :

- Cuidador ansioso e criança voraz: trata-se do modelo clássico e, talvez, o mais frequente. Aqui, a impossibilidade de contenção adequada às necessidades reais do filho, leva a mãe ou cuidador a responder a qualquer estímulo com alimentação o que acarretará, ainda nos primeiros meses de vida, em ganho excessivo de peso. ${ }^{98}$ Esse modelo, entretanto, pode ser transposto para qualquer idade. Dessa forma, são comuns crianças, adolescentes e adultos vorazes e intolerantes à frustração que, ao não encontrarem contenção adequada para suas angústias, utilizam-se do alimento como subterfúgio.

- Pais negligentes: dentro dessa categoria, encontram-se genitores completamente alheios aos cuidados dos filhos. Quase sempre delegam a função a um terceiro, que podem ser avós ou cuidadores, e se esquivam de quaisquer responsabilidades na criação, educação e cuidado dos filhos. Os motivos que levam a tal conduta podem ser os mais variados, indo de depressão, que nesse caso falta condição psíquica para serem cuidadores, até mesmo medo, narcisismo ou execução de tarefas cotidianas, onde o filho é algo que não pode atrapalhar a vida, pois sempre vai existir uma boa razão para que a criança fique em segundo plano.

- Competitividade: a competição entre pai ou mãe e filho ou filha é outro estereótipo comum. Tornar-se pai e mãe exige, além de disponibilidade física, condições psíquicas. O crescimento dos filhos, ao mesmo tempo em que é desejado e bem-vindo, aguça nos pais o sentimento de declínio das próprias condições físicas, acarretando em possível boicote dos genitores, por vezes inconsciente, a tudo o que possa permitir crescer e aflorar dos filhos. Como resultado, qualquer tipo de abordagem, médica, nutricional e psicológica visando emagrecimento torna-se difícil. ${ }^{99}$ 
- Culpa pela ausência: considera-se culpa pela ausência à situação em que os pais, por razões diversas, concretas ou abstratas, como por exemplo, trabalho, compensam seus filhos com comida. Nesse cenário, a criança pode até ter tido regras alimentares cumpridas ao longo do dia ou da semana, mas a chegada do genitor ausente provoca ruptura nas regras e rotinas alimentares. Se as ausências forem frequentes, a falta de regra alimentar será constante. ${ }^{100}$

- Simbiose: nesse quadro, o que une pai e mãe ao filho ou filha, é sempre uma característica comum mantida por ambos, como um pacto velado sendo, no presente caso, a semelhança que interessa é o comer em si, que acaba originando ou mantendo o quadro de obesidade. Assim, a relação se mantém perfeita enquanto o referido pacto não seja rompido por uma das partes. Nesse caso, se quem tentar quebrar o elo for a criança, a mesma sentirá dificuldade maior pois, além de ter que passar por reeducação alimentar, ainda terá que estabelecer nova forma de vínculo com o genitor, suportando os ataques e boicotes constantes do mesmo.

- Sistemas familiares obesogênicos: como o próprio nome indica não se trata de característica ligada a apenas um dos genitores, mas de toda família. Nesse caso, o sistema familiar, como um todo, é comprometido, quando o assunto é alimentação. Todos os membros possuem sobrepeso ou obesidade e a alimentação é sempre desregrada e hipercalórica, ao mesmo tempo em que a prática de atividade física costuma ser desvalorizada. ${ }^{95,101,102}$

- Depressão: ao lado de sintomas mais comuns à depressão, como tristeza, apatia, irritabilidade, isolamento, pode existir, concomitantemente, a perda ou ganho de peso. ${ }^{103}$ Assim, frente a uma criança ou adolescente com sobrepeso/obesidade ou com dificuldades alimentares, a depressão tem que ser investigada e, eventualmente, tratada.

Apesar da complexidade das relações entre obesidade e saúde mental, ${ }^{104}$ aprender a identificar essas combinações ou situações psicossociais permite ao profissional atuar, muitas vezes, diretamente no fator desencadeante ou mantenedor da obesidade, o que resulta em maior probabilidade de êxito no tratamento.

\section{Obesidade somática}

Trata-se de quadro eminentemente ligado ao balanço energético, em que o excesso de tecido adiposo deve-se ao consumo inadequado de calorias frente ao gasto energético ${ }^{105}$. Na anamnese percebe-se história de consumo alimentar excessivo e/ou de sedentarismo. Observam-se os maus hábitos alimentares, como abusos de óleo, açúcar e sal no preparo dos alimentos, ausência de horários para as refeições e simultaneidade de alimentação com outras atividades como assistir à televisão, passeios e reuniões sociais.

$O$ inquérito alimentar quase sempre revela consumo calórico excessivo e a avaliação da atividade física em geral indica sinais de comportamento eminentemente sedentário.

Ocorre, quase sempre, a partir de um momento bem determinado, sendo comum observarse fator desencadeante a partir do qual o balanço energético passou a ser positivo.

Ao exame físico, a criança apresenta aspecto saudável, não se encontrando outros sinais além do panículo adiposo aumentado que, em geral, está bem distribuído ou mais concentrado no abdome. Raramente estão presentes estrias. A pressão arterial tende a ser normal ou pouco elevada. A estatura é pouco elevada ou segue o canal familiar. O perfil lipídico encontra-se quase sempre dentro da normalidade, podendo-se observar, nos casos mais graves, pequenos aumentos nos valores de colesterol ou triglicerídeos, que rapidamente respondem à dietoterapia. A resistência insulínica é frequente apenas nos casos mais graves, com boa resposta ao emagrecimento.

O tratamento é clássico, com associação de dieta hipocalórica, reeducação alimentar e aumento da atividade física, uma vez que na faixa etária pediátrica, as opções farmacológicas para a obesidade somática são praticamente inexistentes. ${ }^{106}$

\section{CONSIDERAÇÕES FINAIS}

Existem várias classificações de obesidade, tais como primária ou secundária (a primária implica em ingestão alimentar excessiva pura, e a secundária na pré-existência de alguma doença que leve ao excesso de peso); exógena, por aumento da ingestão ou endógena, quando estão 
presentes anomalias endócrinas, genéticas, etc. O Código Internacional de Doenças limita-se a dividir obesidade devida a excesso de calorias e obesidade induzida por drogas. Estas classificações têm como base a fisiopatogenia, em última análise, pois discriminam se o aumento de tecido adiposo deve-se ao excesso do alimento ou a outros fatores que levam ao acúmulo de gordura. Há dificuldades de se classificar a obesidade através da etiopatogenia, pois essa escolha torna o atendimento dispendioso, com inúmeras abordagens laboratoriais, as quais nem sempre estão disponíveis na saúde publica ou mesmo privada. Não há dúvida de que a abordagem terapêutica de preferência deverá ser multiprofissional, enfatizando aspectos nutricionais, psicológicos, socioculturais, integrando família e cuidador, uma vez que os tratamentos medicamento e cirúrgico nem sempre serão a primeira escolha.

Sabe-se que, especialmente na área científica, toda classificação sempre será sujeita a críticas. Nesse caso, uma restrição possível à proposta relatada neste artigo é a dificuldade em se caracterizar a resistência periférica à insulina, além de ainda persistirem dúvidas na literatura científica sobre seu papel, havendo tanto relatos de que ela antecede a obesidade como de que ela é apenas mais uma consequência dessa condição.

A classificação apresentada não pretende acrescentar fatos novos ao já extenso, apesar de incompleto, conhecimento sobre a obesidade na infância. Objetiva apenas organizar os conhecimentos existentes, com a finalidade principal de facilitar a tomada de conduta, reforçando a regra cada vez mais aceita de que o tratamento das doenças em geral, e da obesidade em especial, deve ser individualizado.

\section{REFERÊNCIAS BIBLIOGRÁFICAS}

1. Nogueira-de-Almeida CA, Baptista MEC, de Almeida GAN, de Faria Ferraz VE. Obesidade infanto-juvenil: uma proposta de classificação clínica. Pediatr. (São Paulo). 2004;26:257-67.

2. Damiani D. Editorial: Obesidade infanto-juvenil: uma proposta de classificação clínica. Pediatr. (São Paulo). $2004 ; 26: 1$.

3. Nogueira-de-Almeida CA, Mello ED. Nutrologia Pediátrica - Prática Baseada em Evidências: Manole; 2016.

4. Han JC, Lawlor DA, Kimm SY. Childhood obesity. Lancet. $2010 ; 375: 1737-48$.
5. Nishtar S, Gluckman P, Armstrong T. Ending childhood obesity: a time for action. Lancet. 2016; 387:825-7.

6. IBGE. Pesquisa de orçamentos familiares 2008-2009 - Análise do consumo alimentar pessoal no Brasil. In: rendimento Cdte, editor. Rio de Janeiro: IBGE; 2011. p. 150.

7. Ricco RC, Ricco RG, Nogueira-de-Almeida CA, Ramos APP. Comparative study of risk factors among children and adolescents with an anthropometric diagnosis of overweight or obesity. Rev Paul Pediatr. 2010; 28: 320-5.

8. Nogueira-de-Almeida CA, Pires LA, Santos RG. Comparação de indicadores de perfis glicêmico e lipídico entre crianças e adolescentes obesos egressos de serviço público ou privado da cidade de Ribeirão Preto (SP). Medicina (Ribeirão Preto. Online). 2016; 49: 504-10.

9. Nogueira-de-Almeida CA, Garcia J, Caixe SH, Benedeti AC. Ultrasonographic assessment of the common carotid intima-media complex in normal weight children and in overweight/obese children. The FASEB Journal. 2016;30:1165.3-.3.

10. Nogueira-de-Almeida CA, Caixe SH, Benedeti ACGS, Garcia J. Echocardiography evaluation as a marker of cardiovascular risk on obese children and adolescents. The FASEB Journal 2016 30: (Suppl. 1) 126. 1.

11. Nogueira-de-Almeida CA, Benedeti AC, Garcia J, Caixe $\mathrm{SH}$. Correlation between ultrasonographic measures of the abdominal adiposity and indicators of obesity in normal and overweight/obesity children. The FASEB Journal. 2016;30:1165.4-.4.

12. Nogueira-de-Almeida CA, Mello E. Avalliação do estado nutrológico. In: Nogueira-de-Almeida CA, Mello E, editors. Nutrologia Pediátrica: Prática Baseada em Evidências. 1 ed. São Paulo: Manole; 2016. p. 1238.

13. Nogueira-de-Almeida CA, Mello ED. Correlation of body mass index Z-scores with glucose and lipid profiles among overweight and obese children and adolescents. J Pediatr (Rio J). 2018; 94:308-12.

14. Romualdo MC, Nobrega FJ, Escrivao MA. Insulin resistance in obese children and adolescents. J Pediatr (Rio J). 2014;90:600-7.

15. Nogueira-de-Almeida CA, Pinho AP, Ricco RG, Pepato MT, Brunetti IL. Determination of glycemia and insulinemia and the homeostasis model assessment (HOMA) in schoolchildren and adolescents with normal body mass index. J Pediatr (Rio J). 2008; 84: 136-40.

16. Ten S, Maclaren N. Insulin resistance syndrome in children. J Clin Endocrinol Metab. 2004;89:2526-39.

17. Brasil AR. Crianças e adolescentes com sobrepeso ou obesidade: avaliação da reação infalmatória através da dosagem de proteína C reativa ultra sensível e prevalência de síndrome metabólica. Belo Horizonte, 2006; Dissertação (mestrado) - UFMG, Faculdade de Medicina.

18. Cook S, Weitzman M, Auinger $P$, Nguyen $M$, Dietz $\mathrm{WH}$. Prevalence of a metabolic syndrome phenotype in adolescents: findings from the third National Health and Nutrition Examination Survey, 19881994. Arch Pediatr Adolesc Med. 2003;157:821-7.

19. Moran A, Jacobs DR, Jr., Steinberger J, Hong CP, Prineas $R$, Luepker $R$, et al. Insulin resistance during puberty: results from clamp studies in 357 children. Diabetes. 1999; 48:2039-44. 
20. Sinaiko AR, Steinberger J, Moran A, Prineas RJ, Vessby B, Basu $S$, et al. Relation of body mass index and insulin resistance to cardiovascular risk factors, inflammatory factors, and oxidative stress during adolescence. Circulation. 2005; 111:1985-91.

21. Cuartero BG, Lacalle CG, Lobo CJ, Vergaz AG, Rey CC, Villar MJA, et al. Índice HOMA y QUICKI, insulina y peptido $C$ en ninos sanos. Puntos de corte de riesgo cardiovascular. An Pediatr (Barc). 2007; 66: 481-90.

22. Nogueira-de-Almeida CA, Pinho AP, Ricco RG, Pepato MT, Brunetti IL. Determination of glycemia and insulinemia and the homeostasis model assessment (HOMA) in schoolchildren and adolescents with normal body mass index. J Pediatr (Rio J). 2008; 84: $136-40$.

23. Nogueira-de-Almeida CA, de Mello ED. Different Criteria for the Definition of Insulin Resistance and Its Relation with Dyslipidemia in Overweight and Obese Children and Adolescents. Pediatr Gastroenterol Hepatol Nutr. 2018;21:59-67.

24. Pinho AP, Brunetti IL, Pepato MT, Nogueira-deAlmeida CA. Síndrome metabólica em adolescentes do sexo feminino com sobrepeso e obesidade. Rev Paul Pediatr. 2012;30:51-6.

25. Carlone A, Venditti C, Cipolloni L, Zampetti S, Spoletini M, Capizzi M, et al. [Childhhood obesity, insulin resistance and increased cardiovascular risk]. Recenti Prog Med. 2012;103:369-72.

26. Wood P. Insulin Resistance: A Vicious Circle Of Excess Fat. Diabetes in control [Internet]. 2007 June 9th, 2016. Available from: http:// www.diabetesincontrol.com/insulin-resistance-a-vicious-circle- of-excess-fat/ .

27. Kendall D, Vail A, Amin R, Barrett $T$, Dimitri $P$, Ivison $F$, et al. Metformin in obese children and adolescents: the MOCA trial. J Clin Endocrinol Metab. 2013; 98:322-9.

28. Li L, Perez A, Wu LT, Ranjit N, Brown HS, Kelder SH. Cardiometabolic Risk Factors among Severely Obese Children and Adolescents in the United States, 19992012. Child Obes. 2016;12:12-9.

29. Bouchard C. Gene-environment interactions in the etiology of obesity: defining the fundamentals. Obesity (Silver Spring). 2008; 16 Suppl 3:S5-S10.

30. Agurs-Collins T, Bouchard C. Gene-nutrition and gene-physical activity interactions in the etiology of obesity. Introduction. Obesity (Silver Spring). 2008; 16 Suppl 3:S2-4.

31. Wabitsch M, Funcke JB, Lennerz B, Kuhnle-Krahl U, Lahr G, Debatin KM, et al. Biologically inactive leptin and early-onset extreme obesity. N Engl J Med. 2015; 372:48-54.

32. Loos RJ, Bouchard C. FTO: the first gene contributing to common forms of human obesity. Obes Rev. 2008; 9:246-50.

33. Chung WK. An overview of mongenic and syndromic obesities in humans. Pediatr Blood Cancer. 2012; 58: 122-8.

34. Milani D, Cerutti M, Pezzani L, Maffei P, Milan G, Esposito S. Syndromic obesity: clinical implications of a correct diagnosis. Ital J Pediatr. 2014;40:33.

35. Lindgren AC, Barkeling B, Hagg A, Ritzen EM, Marcus C, Rossner S. Eating behavior in Prader-Willi syndrome, normal weight, and obese control groups. J Pediatr. 2000; 137:50-5.
36. Foerste $T$, Sabin M, Reid S, Reddihough D. Understanding the causes of obesity in children with trisomy 21: hyperphagia vs physical inactivity. J Intellect Disabil Res. 2016;60:856-64.

37. Lucas A, Gore SM, Cole TJ, Bamford MF, Dossetor $J F, B a r r$, et al. Multicentre trial on feeding low birthweight infants: effects of diet on early growth. Arch Dis Child. 1984;59:722-30.

38. Desai M, Jellyman JK, Ross MG. Epigenomics, gestational programming and risk of metabolic syndrome. Int J Obes. (Lond). 2015;39:633-41.

39. Williams TC, Drake AJ. What a general paediatrician needs to know about early life programming. Arch Dis Child. 2015; 100: 1058-63.

40. Barker DJ, Eriksson JG, Forsen T, Osmond C. Fetal origins of adult disease: strength of effects and biological basis. Int J Epidemiol. 2002;31:1235-9.

41. Merkestein M, Cagampang FR, Sellayah D. Fetal programming of adipose tissue function: an evolutionary perspective. Mamm Genome. 2014;25:41323.

42. Barker DJ, Winter PD, Osmond C, Margetts B, Simmonds SJ. Weight in infancy and death from ischaemic heart disease. Lancet. 1989;2:577-80.

43. Barker DJ, Osmond C, Kajantie E, Eriksson JG. Growth and chronic disease: findings in the Helsinki Birth Cohort. Ann Hum Biol. 2009;36:445-58.

44. Eriksson JG, Forsen T, Tuomilehto J, Jaddoe VW, Osmond C, Barker DJ. Effects of size at birth and childhood growth on the insulin resistance syndrome in elderly individuals. Diabetologia. 2002;45:342-8.

45. Roszkowska R, Taranta-Janusz K, Wasilewska A. [The role of early-life metabolic programming in the pathogenesis of lifestyle diseases]. Dev Period Med. 2014; 18:477-82.

46. Silveira PP, Portella AK, Goldani MZ, Barbieri MA. Origens desenvolvimentistas da saúde e da doença (DOHaD). J Pediatr (Rio J). 2007;83:494-504.

47. Lane RH. Fetal programming, epigenetics, and adult onset disease. Clin Perinatol. 2014;41:815-31.

48. Segovia SA, Vickers MH, Gray C, Reynolds CM. Maternal obesity, inflammation, and developmental programming. Biomed Res Int. 2014;2014:418975.

49. Hofman $\mathrm{PL}$, Regan $\mathrm{F}$, Jackson WE, Jefferies $\mathrm{C}$, Knight DB, Robinson EM, et al. Premature birth and later insulin resistance. N Engl J Med. 2004; 351:217986.

50. Weber M, Grote V, Closa-Monasterolo R, Escribano J, Langhendries JP, Dain E, et al. Lower protein content in infant formula reduces BMI and obesity risk at school age: follow-up of a randomized trial. Am J Clin Nutr. 2014;99:1041-51.

51. Nogueira-de-Almeida CA, Pires LA, Miyasaka J, Bueno V, Khouri JM, Ramos ML, et al. Comparison of feeding habits and physical activity between eutrophic and overweight/obese children and adolescents: a cross sectional study. Rev Assoc Med Bras (1992). 2015;61:227-33.

52. Piccini RX, Facchini LA, Tomasi E, Thumé E, Silveira DSd, Siqueira FV, et al. Efetividade da atenção prénatal e de puericultura em unidades básicas de saúde do Sul e do Nordeste do Brasil. Rev Bras Saúde Mater Infant. 2007; 7:75-82.

53. Del Ciampo LA, Ricco RG, Daneluzzi JC, Del Ciampo IRL, Ferraz IS, Nogueira-de-Almeida CA. O Programa de Saúde da Família e a Puericultura. Ciênc Saúde Coletiva (online). 2006;11:739-43. 
54. Murphy EF, Clarke SF, Marques TM, Hill C, Stanton $C$, Ross RP, et al. Antimicrobials: Strategies for targeting obesity and metabolic health? Gut Microbes. 2013; 4:48-53.

55. Tremaroli $V$, Backhed F. Functional interactions between the gut microbiota and host metabolism. Nature. 2012;489:242-9.

56. Jess T. Microbiota, antibiotics, and obesity. N Engl J Med. 2014;371:2526-8.

57. Ajslev TA, Andersen CS, Gamborg M, Sorensen TI, Jess T. Childhood overweight after establishment of the gut microbiota: the role of delivery mode, pre-pregnancy weight and early administration of antibiotics. Int J Obes (Lond). 2011;35:522-9.

58. Saari A, Virta LJ, Sankilampi U, Dunkel L, Saxen H. Antibiotic exposure in infancy and risk of being overweight in the first 24 months of life. Pediatrics. 2015; 135:617-26.

59. Dogra S, Sakwinska O, Soh SE, Ngom-Bru C, Bruck WM, Berger B, et al. Dynamics of infant gut microbiota are influenced by delivery mode and gestational duration and are associated with subsequent adiposity. MBio. 2015;6:e02419-14.

60. Schwartz BS, Pollak J, Bailey-Davis L, Hirsch AG, Cosgrove SE, Nau C, et al. Antibiotic use and childhood body mass index trajectory. Int J Obes (Lond). 2016; 40:615-21.

61. Gerber JS, Bryan M, Ross RK, Daymont C, Parks EP, Localio AR, et al. Antibiotic exposure during the first 6 months of life and weight gain during childhood. JAMA. 2016; 315: 1258-65.

62. Cho YM. A gut feeling to cure diabetes: potential mechanisms of diabetes remission after bariatric surgery. Diabetes Metab J. 2014;38:406-15.

63. Suarez-Zamorano N, Fabbiano S, Chevalier C, Stojanovic O, Colin DJ, Stevanovic A, et al. Microbiota depletion promotes browning of white adipose tissue and reduces obesity. Nat Med. 2015;21:1497501.

64. Turnbaugh PJ, Ley RE, Mahowald MA, Magrini V, Mardis ER, Gordon JI. An obesity-associated gut microbiome with increased capacity for energy harvest. Nature. 2006; 444:1027-31.

65. Smith SR. The endocrinology of obesity. Endocrinol Metab Clin North Am. 1996;25:921-42.

66. Han JC, Lawlor DA, Kimm SYS. Childhood obesity. The Lancet. 375:1737-48.

67. Emokpae MA, Adeleke SI, Uwumarongie HO. Subclinical hypothyroidism in childhood obesity and its correlation with lipoproteins. Afr J Med Med Sci. 2011;40:361-5.

68. Min L. Functional Hypercortisolism, Visceral obesity, and metabolic syndrome. Endocr Pract. 2016;22:5068.

69. Germain-Lee EL. Short stature, obesity, and growth hormone deficiency in pseudohypoparathyroidism type 1a. Pediatr Endocrinol Rev. 2006; 3 Suppl 2:31827.

70. Berryman DE, Glad CA, List EO, Johannsson G. The GH/IGF-1 axis in obesity: pathophysiology and therapeutic considerations. Nat Rev Endocrinol. 2013; 9: 346-56.

71. Lizneva D, Suturina L, Walker W, Brakta S, GavrilovaJordan L, Azziz R. Criteria, prevalence, and phenotypes of polycystic ovary syndrome. Fertil Steril. 2016; 106: 6-15.
72. Nezi M, Christopoulos P, Paltoglou G, Gryparis A, Bakoulas V, Deligeoroglou E, et al. Focus on BMI and subclinical hypothyroidism in adolescent girls first examined for amenorrhea or oligomenorrhea. The emerging role of polycystic ovary syndrome. J Pediatr Endocrinol Metab. 2016;29:693-702.

73. Sinai Talaulikar V, Tang T, Yasmin E. Role of metformin in women's health: review of its current place in clinical practice and emerging indications for future. Obstet Gynecol Surv. 2016;71:307-17.

74. Hochberg I, Hochberg Z. Expanding the definition of hypothalamic obesity. Obes Rev. 2010;11:70921.

75. Hochberg I, Hochberg Z. Hypothalamic obesity. Endocr Dev. 2010;17:185-96.

76. Liu J, Zhang A, Li L. Sleep duration and overweight/ obesity in children: review and implications for pediatric nursing. J Spec Pediatr Nurs. 2012;17:193204

77. Woo Baidal JA, Locks LM, Cheng ER, Blake-Lamb $T L$, Perkins ME, Taveras EM. Risk Factors for childhood obesity in the first 1,000 days: A Systematic Review. Am J Prev Med. 2016;50:761-79.

78. Kim TW, Jeong JH, Hong SC. The impact of sleep and circadian disturbance on hormones and metabolism. Int J Endocrinol. 2015;2015:591729.

79. Nogueira-de-Almeida CA, Mello E. Obesidade. In: Nogueira-de-Almeida CA, Mello E, editors. Nutrologia Pediátrica: Prática Baseada em Evidências. $1 \underline{\text { a }}$ ed. São Paulo: Manole; 2016. p. 135-56.

80. Vargas PA. The Link Between Inadequate Sleep and Obesity in Young Adults. Curr Obes Rep. 2016;5:3850 .

81. Cooper SJ, Reynolds GP, With expert c-a, Barnes T, England E, Haddad PM, et al. BAP guidelines on the management of weight gain, metabolic disturbances and cardiovascular risk associated with psychosis and antipsychotic drug treatment. J Psychopharmacol. 2016; 30:717-48.

82. Reekie J, Hosking SP, Prakash C, Kao KT, Juonala $M$, Sabin MA. The effect of antidepressants and antipsychotics on weight gain in children and adolescents. Obes Rev. 2015;16:566-80.

83. Tek C, Chwastiak L. Metformin for weight loss in schizophrenia: safe but not a panacea. Evid Based Ment Health. 2014;17:51-2.

84. Malone M. Medications associated with weight gain. Ann Pharmacother. 2005; 39:2046-55.

85. Choi YJ. Efficacy of adjunctive treatments added to olanzapine or clozapine for weight control in patients with schizophrenia: a systematic review and metaanalysis. The Scientific World Journal. 2015; 2015: 970730 .

86. Whigham LD, Israel BA, Atkinson RL. Adipogenic potential of multiple human adenoviruses in vivo and in vitro in animals. Am J Physiol Regul Integr Comp Physiol. 2006;290: R190-4.

87. Atkinson RL, Dhurandhar NV, Allison DB, Bowen RL, Israel BA, Albu JB, et al. Human adenovirus-36 is associated with increased body weight and paradoxical reduction of serum lipids. Int J Obes (Lond). 2005; 29:281-6.

88. Broderick MP, Hansen CJ, Irvine M, Metzgar D, Campbell K, Baker C, et al. Adenovirus 36 seropositivity is strongly associated with race and gender, but not obesity, among US military personnel. Int j Obes (Lond). 2010; 34:302-8. 
89. Goossens VJ, deJager SA, Grauls GE, Gielen M, Vlietinck RF, Derom CA, et al. Lack of evidence for the role of human adenovirus-36 in obesity in a European cohort. Obesity (Silver Spring). 2011; 19:220-1.

90. Gabbert C, Donohue M, Arnold J, Schwimmer JB. Adenovirus 36 and obesity in children and adolescents. Pediatrics. 2010;126:721-6.

91. Nguyen-Rodriguez ST, Unger JB, Spruijt-Metz D. Psychological determinants of emotional eating in adolescence. Eat Disord. 2009; 17:211-24.

92. Graziano PA, Calkins SD, Keane SP. Toddler selfregulation skills predict risk for pediatric obesity. Int J Obes (Lond). 2010;34:633-41.

93. Bowlby J. Apego e perda: apego, a natureza do vínculo. 2 ed. São Paulo: Martins Fontes; 1990.

94. Anderson SE, Whitaker RC. Attachment security and obesity in US preschool-aged children. Arch Pediatr Adolesc Med. 2011;165:235-42.

95. Almeida CCJN, Mora PO, Oliveira VA, Joao CA, J oao $C R$, Riccio $A C$, et al. Variables associated with family breakdown in healthy and obese/ overweigh adolescents. Rev Paul Pediatr. 2014;32:8.

96. Nogueira-de-Almeida CA, Almeida CCJN, Barreto RF, Oliveira VA, Ferrioli BHVM, João CA, et al. Atuação interdisciplinar em obesidade infanto-juvenil: a experiência do CESNI. Int J Nutrology. 2008;1:7.

97. Almeida CCJN. Psicologia da alimentação. In: Nogueira-de-Almeida CA, Mello E, editors. Nutrologia Pediátrica: Prática Baseada em Evidências. $1 \underline{a}$ ed. São Paulo: Manole; 2016. p. 100-16.
98. Zavaroni DML. Amamentação, sintomas e constituição psíquica. In: Viana TCL, I., editor. Sintomas alimentares, cultura, corpo e obesidade: questoes clínicas e de avaliação: Placebo; 2013.

99. Klein M. Inveja e gratidão: e outros trabalhos 1946 - 1963. Rio de Janeiro: Imago; 1991.

100. Klein M. Amor, culpa e reparação: e outros trabaIhos 1921 - 1945. Rio de Janeiro: I mago; 1996.

101. Araújo MFM, Beserra EP, Araújo TM, Chaves ES. Obesidade infantil: uma reflexão sobre dinâmica familiar numa visão etnográfica. Rev Rene. 2004;7.

102. Bender SC. Influência dos fatores ambientais na obesidade infantil Santa Maria: Centro Universitário Franciscano; 2006.

103. Cruvinel M, Borucho E. Sintomas de depressão infantil e ambiente familiar. Psicol Pesq. 2009;3:87100.

104. Small L, Aplasca A. Child Obesity and Mental Health: A Complex Interaction. Child Adolesc Psychiatr Clin N Am. 2016;25:269-82.

105. Campbell MK. Biological, environmental, and social influences on childhood obesity. Pediatr Res. 2016; 79:205-11.

106. Kelly AS, Fox CK, Rudser KD, Gross AC, Ryder JR. Pediatric obesity pharmacotherapy: current state of the field, review of the literature and clinical trial considerations. Int J Obes (Lond). 2016;40:104350 . 